\title{
Do African American Patients Treated with Radical Cystectomy for Bladder Cancer have Worse Overall Survival? Accounting for Pathologic Staging and Patient Demographics Beyond Race Makes a Difference
}

\author{
Deborah R. Kaye ${ }^{\mathrm{a}, *}$, Joseph K. Canner ${ }^{\mathrm{b}}$, Max Kates ${ }^{\mathrm{a}}$, Mark P. Schoenberg ${ }^{\mathrm{c}}$ \\ and Trinity J. Bivalacqua ${ }^{a}$ \\ ${ }^{a}$ Department of Urology, The James Buchanan Brady Urological Institute, The Johns Hopkins School of Medicine, \\ Baltimore, MD, USA \\ ${ }^{\mathrm{b}}$ Center for Surgical Trials and Outcomes Research, Department of General Surgery, The Johns Hopkins School \\ of Medicine, Baltimore, MD, USA \\ ${ }^{\mathrm{c}}$ Department of Urology, Montefiore Medical Center and Albert Einstein College of Medicine, Bronx, NY, USA
}

\begin{abstract}
.
Background: It is estimated that 74,000 men and women in the United States will be diagnosed with bladder cancer and 16,000 will die from the disease in 2015. The incidence of bladder cancer in Caucasian males is double that of African American males, but African American men and women have worse survival. Although factors contributing to this disparity have been analyzed, there is still great uncertainty as to why this disparity exists.

Objective: To evaluate whether the disparities in bladder cancer survival after radical cystectomy for transitional cell carcinoma (TCC) of the bladder amongst African American (AA) and Caucasian patients is attributable to patient demographics, year of diagnosis, and/or tumor characteristics.

Methods: Using Surveillance, Epidemiology, and End Results Program (SEER) data from 1973-2011, African American and Caucasian patients treated with a radical cystectomy for TCC of the bladder were identified. Primary outcomes were all-cause and cancer-specific mortality. Differences in survival between African Americans and Caucasian patients were assessed using chi-square tests for categorical variables and Student's $t$-tests for continuous variables. Cox proportional hazards regression was used to measure the hazard ratio for African Americans compared to Caucasians for all-cause and cancer-specific mortality. In addition, coarsened matching techniques within narrow ranges, were used to match African American and Caucasian patients on the basis of age, sex, and cancer stage. Following matching, differences in all-cause and cancer-specific mortality were again assessed using a stratified Cox proportional hazards model, using the matching strata for the regression strata.
\end{abstract}

\footnotetext{
*Correspondence to: Deborah R. Kaye, The James Buchanan Brady Urological Institute, The Johns Hopkins School of Medicine, 600 N. Wolfe Street, Marburg 401, Baltimore, MD
} 
Results: The study cohort consisted of 21,406 African American and Caucasian patients treated with radical cystectomy for bladder urothelial cancer, with $6.2 \%$ being African American and 73.9\% male. African American patients had worse all-cause and cancer-specific mortality in the univariable analysis (all-cause: HR: 1.23 ; 95\% CI 1.15-1.32, $p<0.001$ ); bladder-cancer specific: HR 1.21; 95\% CI 1.11-1.33; $p<0.001)$. However, after accounting for sex, age, year of diagnosis, marital status, region of treatment, and stage at cystectomy, all-cause mortality was significant (HR 1.20; 95\% CI 1.12-1.29; $p<0.0001$ ), but not bladder-cancer specific mortality (HR 1.09; 95\% CI 1.00-1.20; $p<0.053$ ). Predictors of bladder cancer specific mortality were age, sex, stage of disease, and marital status. The matched analysis yielded a roughly $1: 15$ match, with 22,511 Caucasians being matched to 1,509 African American patients. In the matched analysis, African Americans had increased all-cause mortality (HR 1.17; 95\% CI 1.09-1.26; $p<0.0001$ ), but bladder-cancer specific mortality was no longer significant (HR 1.08; 95\% CI 0.99-1.18; $p<0.102)$.

Conclusions: African Americans who undergo a cystectomy are more likely to die, but not necessarily solely because of bladder cancer. Although African American patients have worse all-cause and cancer-specific mortality in univariable models, after controlling for sex, age, year of diagnosis, marital status, region of treatment, and stage at cystectomy, African American patients still have worse overall survival, but equivalent bladder-cancer specific survival. Differences in age, sex, and stage at diagnosis explain some, but not all of the differences in survival.

Keywords: Urothelial cancer, racial disparities, cystectomy

\section{INTRODUCTION}

It is estimated that 74,000 men and women in the United States will be diagnosed with bladder cancer and 16,000 will die from the disease in 2015 [1]. Transitional cell carcinoma of the bladder accounts for $>90 \%$ of bladder cancers in the United States. Risk factors include smoking and environmental exposure to tobacco, occupational exposure to carcinogens, including aromatic amines, aniline dyes, and cyclophosphamide, arsenic pollution in drinking water, and radiation exposure. Genetic susceptibility also plays a role in the incidence, prevalence and mortality of the disease [2]. Presenting signs and symptoms include hematuria and irritative voiding symptoms. Mortality is determined by initial tumor stage, tumor characteristics, response to treatment and patient co-morbidities.

Even though the incidence of bladder cancer in Caucasian males is double that of African American males, African American men and women have worse survival $[3,4]$. Although some differences in survival may be attributable to worse stage at presentation, a number of studies have demonstrated worse disease-specific survival amongst African Americans even after controlling for demographic factors, stage and treatment [3, 5-9]. Other proposed explanations for the decreased cancer-specific survival amongst African American patients with bladder cancer are differences in smoking incidence and occupational exposures, unequal access to health care, delays in seeking treatment, marital status, and differences in quality and type of care [2].
Although these factors have been analyzed, there is still great uncertainty as to why racial disparities exist in survival among patients treated with cystectomy for bladder cancer. For this reason, we performed a population-based analysis to demonstrate the differences in overall and bladder cancer-specific survival and factors that may affect the disparate outcomes. We further attempted to decrease the uncertainty by performing a matched analysis - matching individual African American patients who had undergone a cystectomy to similar Caucasian patients based on age, sex, and stage, and exploring whether, even when patients were matched on these characteristics, if the differences in overall and bladder-cancer specific mortality remain. While other studies have described differences in survival amongst African American and Caucasian patients with bladder cancer, this study is unique in that it uses SEER data to analyze only those patients who underwent a cystectomy for treatment and explores if differences in survival remain when patients are matched on age, sex, and tumor stage.

\section{METHODS}

The Surveillance, Epidemiology, and End Results (SEER) Program of the National Cancer Institute (NCI) was instituted in 1973 to collect data on cancer cases from various locations throughout the United States. As of the November 2013 data submission, SEER contained records for 7.4 million cases of malignant cancer from 18 sentinel registries covering a population of 86 million people. 
We queried the SEER database to obtain all cases of malignant cancer in the urinary bladder (ICD-O-3 codes C67.0-C67.9). This resulted in 323,800 cases from 1973-2011. We extracted demographic variables (age, race, sex, region, insurance status, and marital status), information about the disease (year of diagnosis, cancer stage, number of primaries) and treatments received, and vital status, including time to death and cause of death. In situations where there were multiple records for the same patient (4.7\% of cases), only the most recent record was retained, although information on previous partial cystectomies was obtained. Patients were included in the study if they were age 40 or older, of Caucasian or African American race, and underwent a total cystectomy. There were 24,130 patients meeting these criteria. We only included patients who had data for all parameters. Cancer staging data is only available starting in 1998; excluding patients with missing cancer stage results in a study cohort of 21,406.

Differences between Caucasians and African Americans and between those who died and survived were assessed using chi-square tests for categorical variables and Student's $t$-tests for continuous variables. Cox proportional hazards regression was used to measure the differences in all-cause and cancerspecific mortality for African Americans compared to Caucasians. This difference is expressed as a hazard ratio (HR). In addition, coarsened exact matching techniques were used to match Caucasian and African American patients on the basis of age, sex, and cancer stage. Age was coarsened into bins of width four years, while sex and cancer stage were matched exactly. Following matching, a stratified Cox proportional hazards model was fit, using the matching strata for the regression strata. Since many-to-one matching was used, the Cox model was also weighted according to the number of Caucasian patients matched to each African American patient.

All analyses were performed using Stata 12.1 (StataCorp, College Station, Texas). The user-written Stata command cem was used to perform coarsened exact matching [10]. The study was exempt from IRB approval because the data did not contain unique identifiers.

\section{RESULTS}

The study cohort included 21,406 African American and Caucasian patients treated with radical cystectomy for urothelial cancer. Table 1 shows demographic and clinical characteristics by race. Of all patients in the study cohort, $6.2 \%$ were African American, $73.9 \%$ were male, and the mean age was 67.9 years. Overall, nearly two-thirds of patients (65.9\%) were diagnosed between age 60-79.

African Americans had a lower mean age at diagnosis than Caucasians (64.5 vs. $67.9 ; p<0.001)$ and the distribution of age at diagnosis was significantly different between African Americans and Caucasians $(p<0.001)$. For example, a higher proportion of African American patients were diagnosed between 50-59 years than their Caucasian counterparts (24.2\% vs $16.3 \%)$; conversely more Caucasian patients were diagnosed between 70-79 years than African American patients $(34.7 \%$ vs 27.6\%). African American patients were less likely to be male than Caucasian patients (59.8\% vs. $74.9 \%$; $p<0.001)$. More African American patients had metastatic disease at time of cystectomy $(39.8 \%$ vs $32.1 \%$ ) and the difference in stage at cystectomy was statistically significant $(p<0.001)$. African American patients were slightly more likely to have only one primary malignancy $(58.4 \%$ vs $54.1 \%, p=0.011)$.

More Caucasian patients than African American patients were married $(65.8 \%$ vs $42.2 \%, p<0.001)$ and a greater proportion of Caucasian patients lived in the Pacific and Southwest regions, while more African American patients lived in the East and North regions $(p<0.001)$. African Americans and Caucasians had a similar distribution of year of diagnosis.

\section{Unmatched analysis}

As shown in Fig. 1 and Table 2a, in the univariable unmatched analysis, African Americans had worse all-cause mortality (HR 1.23 ; 95\% CI $1.15-1.32$; $p<0.001$ ) and cancer-specific mortality (HR 1.23; 95\% CI $1.13-1.34 ; p<0.001)$ than Caucasians. Table $2 \mathrm{a}$ also shows the results for the unmatched multivariable analysis of all-cause mortality and Table $2 \mathrm{~b}$ shows bladder cancer-specific mortality. In the multivariable analysis, African Americans had worse all-cause mortality (HR 1.20; 95\% CI $1.12-1.29 ; p<0.001)$, and slightly, but not statistically significantly, higher bladder cancer-specific mortality (HR 1.09; 95\% CI 1.00-1.20; $p=0.053$ ). Higher stage at cystectomy, not being married, and residing in regions other than the Pacific Coast were associated with an increased risk of both all-cause mortality and cancer-specific mortality. Males had equivalent risk of all-cause mortality to females (HR 
Table 1

Demographics by race

\begin{tabular}{|c|c|c|c|c|}
\hline & $\begin{array}{c}\text { AA }(\%) \\
N=1,328 \\
\end{array}$ & $\begin{array}{c}\text { Caucasian (\%) } \\
N=20,078\end{array}$ & $\begin{array}{c}\text { Total }(\%) \\
N=21,406 \\
\end{array}$ & $P$-value \\
\hline \multicolumn{5}{|l|}{ Age } \\
\hline $40-49$ & 9.0 & 4.9 & 5.1 & \multirow[t]{5}{*}{$<0.001$} \\
\hline $50-59$ & 24.2 & 16.3 & 16.8 & \\
\hline $60-69$ & 31.8 & 31.5 & 31.6 & \\
\hline $70-79$ & 27.6 & 34.7 & 34.3 & \\
\hline $80+$ & 7.4 & 12.6 & 12.3 & \\
\hline Age at Diagnosis, Mean (SD) & $64.5(10.7)$ & $67.9(10.2)$ & $67.9(10.2)$ & $<0.001$ \\
\hline Male $(\%)$ & 59.8 & 74.9 & 73.9 & $<0.001$ \\
\hline \multicolumn{5}{|l|}{ Year of Diagnosis } \\
\hline 1988-1990 & 3.0 & 3.8 & 3.7 & \multirow[t]{5}{*}{0.060} \\
\hline 1991-1995 & 8.8 & 10.5 & 10.4 & \\
\hline 1996-2000 & 17.4 & 15.6 & 15.8 & \\
\hline 2001-2005 & 31.2 & 29.7 & 29.8 & \\
\hline 2006-2011 & 39.5 & 40.4 & 40.4 & \\
\hline Year of Diagnosis, Mean (SD) & $2003(6)$ & $2003(6)$ & $2003(6)$ & 0.330 \\
\hline Married (\%) & 42.2 & 65.8 & 64.3 & $<0.001$ \\
\hline \multicolumn{5}{|l|}{ Region } \\
\hline East & 44.4 & 31.6 & 32.4 & \multirow[t]{4}{*}{$<0.001$} \\
\hline North & 18.4 & 15.4 & 15.6 & \\
\hline Pacific & 36.3 & 47.6 & 46.9 & \\
\hline Southwest & 1.0 & 5.4 & 5.1 & \\
\hline \multicolumn{5}{|l|}{ Stage at Cystectomy } \\
\hline Ois, Oa, I & 11.0 & 12.6 & 12.5 & \multirow[t]{3}{*}{$<0.001$} \\
\hline II-III & 49.3 & 55.3 & 54.9 & \\
\hline IV & 39.8 & 32.1 & 32.5 & \\
\hline \multicolumn{5}{|l|}{ Tumor Count } \\
\hline 1 & 95.4 & 94.2 & 94.2 & \multirow[t]{3}{*}{0.160} \\
\hline 2 & 4.5 & 5.8 & 5.7 & \\
\hline $3+$ & 0.1 & 0.1 & 0.1 & \\
\hline \multicolumn{5}{|l|}{ Number of Primary Malignancies } \\
\hline 1 & 58.4 & 54.1 & 54.4 & \multirow[t]{3}{*}{0.011} \\
\hline 2 & 37.8 & 41.7 & 41.5 & \\
\hline $3+$ & 3.8 & 4.2 & 4.2 & \\
\hline
\end{tabular}

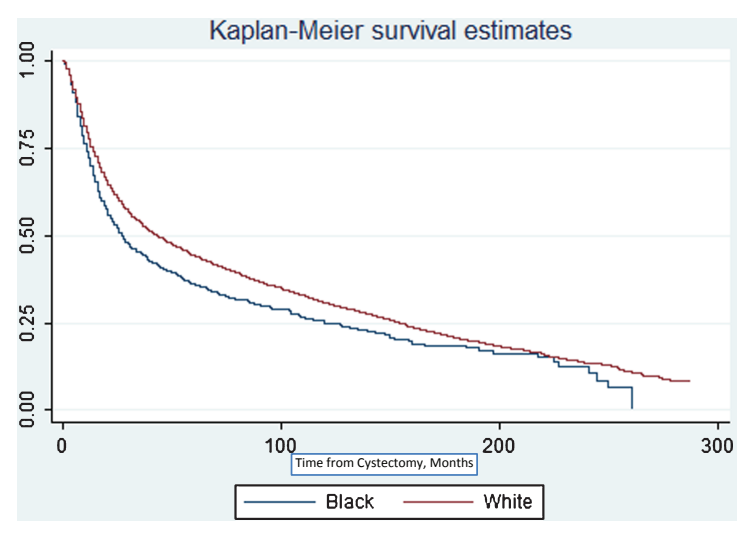

Fig. 1. Unmatched all-cause mortality.

$1.00 ; 95 \%$ CI $0.96-1.04 ; p=0.93)$, but were at lower risk of cancer-specific mortality (HR $0.85 ; 95 \%$ CI $0.81-0.89 ; p<0.001)$.

\section{Matched analysis}

African Americans were matched to Caucasian patients on age, sex, and cancer stage, with 19,975 Caucasians matched to 1,328 African American patients, on average a 15:1 match. There were matches for all African American patients and only 103 Caucasian patients were not matched. The cohorts were well matched, based on the L1 statistic of 0.11 . After matching there were no differences between Caucasians and African Americans with respect to age, sex, cancer stage, year of diagnosis, number of tumors and number of primary malignancies. Table $3 \mathrm{a}$ shows the results for the matched all-cause mortality analysis and Table $3 \mathrm{~b}$ shows the results for the matched bladder cancer-specific mortality analysis. In the univariable analysis, matching slightly increased the hazard ratio for all-cause mortality among African Americans to 1.24 (95\% 
Table 2a

Unmatched all-cause mortality

\begin{tabular}{lcccr}
\hline & HR & CI & & $P$ Value \\
\hline $\begin{array}{l}\text { Univariable } \\
\text { AA Race }\end{array}$ & 1.23 & 1.15 & 1.32 & $<0.01$ \\
$\begin{array}{l}\text { Multi-variable } \\
\text { Black race }\end{array}$ & 1.20 & 1.12 & 1.29 & $<0.01$ \\
Male & 1.00 & 0.96 & 1.04 & 0.99 \\
Age & & & & \\
40-45 & & & & \\
45-49 & 1.23 & 1.00 & 1.50 & 0.05 \\
50-54 & 1.23 & 1.01 & 1.49 & 0.04 \\
55-59 & 1.34 & 1.11 & 1.61 & $<0.01$ \\
60-64 & 1.59 & 1.33 & 1.91 & $<0.01$ \\
65-69 & 1.82 & 1.52 & 2.17 & $<0.01$ \\
70-74 & 2.15 & 1.79 & 2.57 & $<0.01$ \\
75-79 & 2.52 & 2.11 & 3.02 & $<0.01$ \\
80-84 & 3.20 & 2.67 & 3.85 & $<0.01$ \\
85+ & 3.90 & 3.19 & 4.77 & $<0.01$ \\
Year of Diagnosis & & & & \\
1988-1990 & & & & \\
1991-1995 & 1.04 & 0.95 & 1.14 & 0.36 \\
1996-2000 & 1.03 & 0.94 & 1.12 & 0.52 \\
2001-2005 & 1.07 & 0.99 & 1.17 & 0.10 \\
$\quad$ 2006-2011 & 1.01 & 0.93 & 1.10 & 0.84 \\
Married & 0.81 & 0.77 & 0.83 & $<0.01$ \\
Region & & & & \\
East & & & & \\
Northeast & 0.98 & 0.90 & 1.00 & 0.04 \\
Pacific Coast & 0.86 & 0.83 & 0.90 & $<0.01$ \\
$\quad$ Southwest & 1.01 & 0.93 & 1.09 & 0.82 \\
Stage at Cystectomy & & & & \\
$\quad$ I II,II & & & & \\
IV & 1.50 & 1.41 & 1.60 & $<0.01$ \\
\hline & 3.41 & 3.20 & 3.65 & $<0.01$ \\
\hline
\end{tabular}

CI 1.16-1.33; $p<0.001)$, and decreased the hazard ratio for cancer-related mortality to $1.13(95 \% \mathrm{CI}$ $1.04-1.24 ; p=0.006$ ).

As in the unmatched multivariable analysis, in the matched multivariable analysis, African American patients had worse all-cause mortality than Caucasians (HR 1.17; 95\% CI 1.09-1.26; $p<0.001$ ). However, the risk of bladder cancer specific mortality for African Americans was no longer significantly higher than that for Caucasians (HR 1.08; 95\% CI $0.99-1.18 ; p=0.102)$. As in the unmatched analysis, not being married and residing in areas other than the Pacific Coast region were associated with an increased risk of all-cause mortality. In addition, being diagnosed between 2001 and 2005 was associated with an increased risk of all-cause mortality. Similarly, not being married, residing in the Pacific Coast region, and being diagnosed between 2001 and 2011 were associated with an increased risk of bladder cancer-specific mortality.
Table $2 b$

Unmatched bladder-cancer specific mortality

\begin{tabular}{|c|c|c|c|c|}
\hline & HR & $\mathrm{CI}$ & & $P$ Value \\
\hline \multicolumn{5}{|l|}{ Univariable } \\
\hline AA Race & 1.21 & 1.11 & 1.33 & $<0.01$ \\
\hline \multicolumn{5}{|l|}{ Multi-variable } \\
\hline AA race & 1.09 & 1.00 & 1.20 & 0.053 \\
\hline Male & 0.85 & 0.81 & 0.89 & $<0.01$ \\
\hline \multicolumn{5}{|l|}{ Age } \\
\hline \multicolumn{5}{|l|}{$40-45$} \\
\hline $45-49$ & 1.16 & 0.92 & 1.46 & 0.213 \\
\hline $50-54$ & 1.16 & 0.93 & 1.45 & 0.184 \\
\hline $55-59$ & 1.17 & 0.95 & 1.45 & 0.148 \\
\hline $60-64$ & 1.31 & 1.06 & 1.61 & 0.012 \\
\hline $65-69$ & 1.42 & 1.15 & 1.75 & $<0.01$ \\
\hline $70-74$ & 1.50 & 1.22 & 1.84 & $<0.01$ \\
\hline $75-79$ & 1.69 & 1.37 & 2.08 & $<0.01$ \\
\hline $80-84$ & 2.15 & 1.74 & 2.66 & $<0.01$ \\
\hline $85+$ & 2.34 & 1.84 & 1.84 & $<0.01$ \\
\hline \multicolumn{5}{|c|}{ Year of Diagnosis } \\
\hline \multicolumn{5}{|c|}{ 1988-1990 } \\
\hline 1991-1995 & 1.08 & 0.95 & 1.23 & 0.21 \\
\hline 1996-2000 & 1.09 & 0.97 & 1.23 & 0.15 \\
\hline 2001-2005 & 1.18 & 1.05 & 1.33 & 0.01 \\
\hline 2006-2011 & 1.11 & 0.99 & 1.26 & 0.07 \\
\hline Married & 0.85 & 0.81 & 0.89 & $<0.01$ \\
\hline \multicolumn{5}{|l|}{ Region } \\
\hline \multicolumn{5}{|l|}{ East } \\
\hline Northeast & 0.96 & 0.89 & 1.03 & 0.24 \\
\hline Pacific Coast & 0.90 & 0.85 & 0.94 & $<0.01$ \\
\hline Southwest & 1.03 & 0.93 & 1.14 & 0.58 \\
\hline \multicolumn{5}{|c|}{ Stage at Cystectomy } \\
\hline \multicolumn{5}{|c|}{ I } \\
\hline II,III & 2.13 & 1.92 & 2.36 & $<0.01$ \\
\hline IV & 6.10 & 5.50 & 6.77 & $<0.01$ \\
\hline Married & 0.85 & 0.82 & 0.90 & $<0.01$ \\
\hline \multicolumn{5}{|l|}{ Region } \\
\hline \multicolumn{5}{|l|}{ East } \\
\hline Northeast & 0.96 & 0.89 & 1.03 & 0.24 \\
\hline Pacific Coast & 0.90 & 0.85 & 0.94 & $<0.01$ \\
\hline Southwest & 1.03 & 0.93 & 1.14 & 0.58 \\
\hline \multicolumn{5}{|c|}{ Stage at Cystectomy } \\
\hline \multicolumn{5}{|c|}{ I } \\
\hline II,III & 2.13 & 1.92 & 2.36 & $<0.01$ \\
\hline IV & 6.10 & 5.50 & 6.77 & $<0.01$ \\
\hline
\end{tabular}

\section{DISCUSSION}

Even though the incidence of bladder cancer in Caucasian males is double that of African American males, African American men and women have worse survival, even after controlling for demographic factors, stage and treatment [3-9]. In order to decrease health care disparities, it is critical to understand in what specific populations disparities exist and what factors may be driving the differences in outcomes. Our analysis suggests that although African American patients who undergo a cystectomy for bladder cancer have worse all-cause and cancer-specific mortality than Caucasians. The racial 
Table 3a

Matched all-cause mortality

\begin{tabular}{lcccr}
\hline & HR & CI & & $P$ Value \\
\hline $\begin{array}{l}\text { Univariable } \\
\text { Race }\end{array}$ & 1.24 & 1.16 & 1.33 & $<0.01$ \\
$\begin{array}{l}\text { Multi-variable } \\
\text { Black race }\end{array}$ & 1.17 & 1.09 & 1.26 & $<0.01$ \\
$\begin{array}{l}\text { Year of Diagnosis } \\
\quad \text { 1988-1990 }\end{array}$ & & & & \\
$\quad$ 1991-1995 & 1.08 & 0.99 & 1.18 & 0.08 \\
1996-2000 & 1.04 & 0.96 & 1.14 & 0.32 \\
2001-2005 & 1.13 & 1.04 & 1.23 & $<0.01$ \\
$\quad$ 2006-2011 & 1.07 & 0.98 & 1.17 & 0.11 \\
Married & 0.79 & 0.76 & 0.82 & $<0.01$ \\
Region & & & & \\
$\quad$ East & & & & \\
$\quad \begin{array}{l}\text { Northeast } \\
\text { Pacific Coast }\end{array}$ & 0.94 & 0.89 & 1.00 & 0.03 \\
$\quad$ Southwest & 1.01 & 0.81 & 0.88 & $<0.01$ \\
& & 0.93 & 1.09 & 0.90 \\
\hline
\end{tabular}

Table $3 b$

Matched bladder-cancer specific mortality

\begin{tabular}{lcccr}
\hline & HR & CI & & $P$ Value \\
\hline $\begin{array}{l}\text { Univariable } \\
\text { Race }\end{array}$ & 1.13 & 1.04 & 1.24 & $<0.01$ \\
$\begin{array}{l}\text { Multi-variable } \\
\text { Black race }\end{array}$ & 1.08 & 0.99 & 1.18 & 0.10 \\
$\begin{array}{l}\text { Year of Diagnosis } \\
\quad \text { 1988-1990 }\end{array}$ & & & & \\
$\quad 1991-1995$ & 1.12 & 0.99 & 1.26 & 0.07 \\
1996-2000 & 1.10 & 0.98 & 1.24 & 0.10 \\
2001-2005 & 1.24 & 1.11 & 1.38 & $<0.01$ \\
$\quad$ 2006-2011 & 1.16 & 1.04 & 1.30 & 0.01 \\
Married & 0.84 & 0.80 & 0.88 & $<0.01$ \\
Region & & & & \\
$\quad$ East & & & & \\
$\quad$ Northeast & 0.98 & 0.91 & 1.04 & 0.47 \\
$\quad \begin{array}{l}\text { Pacific Coast } \\
\text { Southwest }\end{array}$ & 0.89 & 0.84 & 0.93 & $<0.01$ \\
\hline
\end{tabular}

disparity in bladder-cancer specific survival after radical cystectomy can be explained by adjusting for sex, age, year of diagnosis, marital status, region of treatment, and stage of disease at time of cystectomy. However, these variables do not explain all of the differences in all-cause mortality.

In the unmatched univariable analysis, African American patients have a $23 \%$ increased risk of all-cause mortality, which decreases to $20 \%$ in the multivariable model. In contrast, African Americans have a $21 \%$ increased risk of bladder cancer-specific mortality, but after controlling for the above variables, the remaining $9 \%$ difference is no longer significant. Advanced age, not being married, residing in areas other than the Pacific region, and later stages of disease were associated with worse all-cause mortality as well as with bladder cancer-specific mortality.
Women were at increased risk for bladder-cancer specific mortality but not all-cause mortality.

Matching based on age, sex, and stage of cancer at time of radical cystectomy generally had little effect on the multivariable analysis results. These findings suggest that sex, age, and stage at cystectomy are important variables explaining some of the differences in both all-cause and bladder cancer-specific mortality, but they do not fully account for the survival differences. In addition, marital status seems to play an important role in explaining mortality differences between African Americans and Caucasians. Notably, in both unmatched and matched multivariable analyses, the risk of bladder cancer-specific mortality for African Americans was no longer significantly higher than that for Caucasians. This suggests that African American patients may be dying from causes not directly related to the diagnosis and management of their bladder cancer. Several reasons have been cited as contributing to the increased mortality rate amongst African Americans who have undergone a cystectomy for bladder cancer, some of which are consistent with our findings and some of which we are unable to assess using the SEER dataset.

\section{Cancer stage \& grade}

Histologic type, stage and grade have been shown to affect differences in African American and Caucasian bladder cancer survival [11]. African American patients have been found to have a higher stage and grade, and greater extent of disease at diagnosis $[7,12,13]$. In our analysis, patients with muscle invasive disease had over 2 times the risk of bladdercancer mortality than those who were diagnosed with bladder cancer at lower stages. Those with distant disease had nearly three times the risk of all-cause and six times the risk of bladder-cancer specific mortality. However, stage did not fully account for differences in survival between the cohorts. Controlling for stage, age, and sex decreased the risk of all-cause mortality by $10 \%$ and bladder-cancer by $8 \%$, but African Americans still had worse all-cause mortality.

Using a population-based tumor registry, Prout and colleagues demonstrated that African American patients were more likely to present with muscle invasive disease and that African American patients with $\mathrm{T} 2$ and $\mathrm{T} 3$ urothelial cancer had worse survival compared to Caucasians. However, there was no survival difference in patients with non-muscle invasive or advanced disease. They also found that there were no differences in diagnostic tests, transurethral resection 
rates, or the use of radiation and chemotherapy [14]. In an analysis using SEER data from 1993-1999, Lee and colleagues demonstrated that African American patients present with a higher proportion of highgrade tumors than Caucasian patients [15]. These findings were confirmed in an analysis of the National Cancer Data Base, where Mallin, et al. demonstrated that African American patients, especially African American females had a lower proportion of stage 0 tumors and a higher proportion of high-grade tumors [3].

Yee and colleagues demonstrated that even when controlling for stage and grade, African Americans had worse five year disease-specific survival compared to Caucasians, Hispanics, and Asian/Pacific Islanders (70.2\% in African Americans compared to $82.8 \%, 80.7 \%$, and $81.9 \%$ in Caucasians, Hispanics, and Asian/Pacific Islanders respectively). Congruent to our analysis, Scosyrev, et al. showed that adjusting for age, sex and grade only eliminated $50-70 \%$ of the excess hazard of death from bladder cancer amongst African Americans. The authors concluded that other factors, such as host factors, differing choices and efficacies of treatments, and differences within a tumor group were responsible for the remaining differences in hazard ratios [9].

\section{Marital status}

Marital status is an important predictor of allcause and bladder cancer specific mortality in all of our analyses. Married individuals, both African American and Caucasian, have decreased overall and bladder-cancer specific mortality. Since Caucasian patients are more often married than African Americans, including married in the model removes some of the disparity. Similar findings have been realized in other studies, where marital status has been found to be a predictor of cancer specific survival in bladder cancer and a number of other disease states $[16,17]$. Results from the National Longitudinal Mortality Study showed that marriage conferred a survival benefit for patients in all disease states [18]. In a SEER-Medicare study of 19,982 bladder cancer patients, Datta and colleagues demonstrated that irrespective of stage, married men had better survival than unmarried men independent of age, race, socioeconomic status, or co-morbidities. In contrast, co-morbid conditions and age explained the survival difference in women with stages II-IV bladder cancer [19]. Another SEER study only found an increased risk of death to widowed men of 1.74 relative to married men after being diagnosed with bladder cancer (95\% CI 1.15, 2.26, $P=0.008$ ) [20]. Gore, et al. used SEER data to evaluate the role of marital status on survival amongst patients undergoing radical cystectomy for bladder cancer and found that married subjects had significantly better survival than did single or widowed subjects $(P<0.001)$ and trended towards improved survival compared to separated/divorced subjects $(P=0.20)[17]$. The authors hypothesized that some of the differences in survival could be secondary to differences in cancer screening and disease detection, risk behaviors, access to care, and the interplay between psychosocial factors and one's immune system.

\section{Age and sex}

Age was correlated with increased all-cause and bladder cancer specific mortality. In an analysis of a population-based cancer registry, Noon and colleagues found that bladder cancer specific mortality was highly correlated with age and that bladder cancer specific mortality was highest among the elderly [21]. In an analysis of 31,100 patients with bladder cancer in Florida, Brookfield, et al. demonstrated that older age was predictive of poorer outcomes [4]. Mallin, and colleagues showed that older age had the highest hazard ratios for bladder-cancer specific mortality [3].

In our analysis, females had a $15 \%$ increased risk of bladder cancer specific mortality compared to males, but no increased risk of all-cause mortality. Noon, et al found that females with high risk bladder cancer had worse bladder cancer specific mortality, but not all-cause mortality. There were no significant differences in 5 year all-cause of bladder-cancer specific mortality for patients with low-risk or muscle invasive bladder cancer [21]. In a propensity score matching analysis, Choo, et al found that female gender was not an independent risk factor for overall survival or bladder cancer specific survival [22]. Mallin, et. al showed that, after controlling for demographic and clinical differences, in early stage disease, Caucasian females had significantly lower mortality then Caucasian males, but for late-stage disease African American males (HR 1.3; CI 1.2-1.4) and African American females (HR 1.3; CI 1.2-1.3) had increased mortality to Caucasian males, but not to Caucasian females [3].

The finding that African Americans had a lower mean age at diagnosis than Caucasians (64.5 vs. 67.9; $p<0.001)$ and that the distribution of age at diagnosis 
was significantly different between African Americans and Caucasians $(p<0.001)$ is interesting and contrary to what would be expected when examining smoking rates in African Americans and Caucasians. Although overall smoking rates are similar amongst African Americans and Caucasians, African American men are more likely to smoke than Caucasian men, but African American women are less likely to smoke than Caucasian women [23]. In addition, Caucasian women start smoking at an earlier age than African American women [24]. When examining smoking rates, by age, smoking rates for Caucasian women are higher than for African American women at each age group, except for in the mid 30's to late 40 's, where smoking rates are higher for African American women than Caucasian women.

\section{Regional variation}

Underwood and colleagues found that patients who were diagnosed in Iowa or Detroit were more likely to die of bladder cancer than those diagnosed in Utah or New Mexico [5]. In Atlanta, African American patients were more likely to die from bladder cancer than similar Caucasian patients and also had increased bladder-cancer specific mortality than African American patients diagnosed in all other regions except Atlanta. Our analysis demonstrated regional variation in bladder cancer and overall mortality. Patients diagnosed in the Pacific Coast region had a lower risk of all-cause and bladder cancerspecific mortality than those diagnosed in the East.

\section{Treatment differences}

This analysis demonstrates that there are no African American/Caucasian racial disparities in bladder cancer-specific survival amongst patients who undergo a cystectomy for treatment of bladder cancer after controlling for other variables. This suggests that African American and Caucasian patients who undergo a cystectomy have equal responses to therapy and get equivalent treatment. Patients who do not get treated with a cystectomy may have additional barriers to care or differences in type, quality, or intensity of treatment, which are not examined in this analysis. Other studies have determined that disparities in survival could be secondary to treatment differences, such as African American patients being more likely to receive no treatment [25], radiation alone [26], or radiation and chemotherapy [11]. In contrast, a study of SEER-Medicare patients with early stage bladder cancer, Hollenbeck et al. determined that the racial disparity in bladder cancer mortality was not due to differences in treatment intensity or quality of care. In fact, African American patients were more likely to undergo a restaging resection $(12 \%$ vs $6.5 \% ; p<0.01)$ and urine cytology (36.8\% vs $29.7 \% ; p<0.01)$ [27]. Proust also found no racial disparities in treatment [14].

Several authors have shown racial differences in location of treatment and short-term outcomes after undergoing a cystectomy. Using Healthcare Cost and Utilization Project State Inpatient Databases, Barocas and colleagues determined that African American patients who had a radical cystectomy were treated more often at a low volume center and by low volume surgeons compared to Caucasian patients. This finding was confirmed by Casey, et al. who demonstrated that African American race was associated with a higher likelihood of receiving care at a low volume or very low volume hospital [28]. In addition, African American patients had a pelvic lymphadenectomy less frequently and their surgery was associated with more adverse events, including inpatient hospital mortality, than Caucasian patients [29]. Konety et al had similar findings with regards to African American patients being least likely to undergo a radical cystectomy at a high/medium volume hospital. After controlling for confounding variables, African American patients had an increased risk of complications and longer length-of-stays, but no differences in in-hospital mortality [30]. Taub, et al. used the Nationwide Inpatient Sample to determine that even after adjusting for confounders, African Americans were more likely to have an in-hospital mortality and longer length of stay [31].

\section{Co-morbidities}

Patients with bladder cancer frequently have comorbidities, which can influence treatment options, including limiting the use of neoadjuvant/adjuvant chemotherapy and/or surgery as well as increasing complications. Co-morbidity is a significant predictor of overall death in patients with both non muscle- and muscle- invasive bladder cancer [13, 32]. In a Danish-population based cohort study of 3,997 patients with muscle-invasive bladder cancer, $43 \%$ of patients had at least one co-morbidity and mortality rates were higher amongst patients with more co-morbidities. The authors also showed that co-morbidities increased over the 12 year study period [33]. Using a multi-institutional database 
of patients treated with a radical cystectomy for bladder cancer in Alberta, Canada, Fairey and colleagues demonstrated that increased co-morbidity was associated with worse overall survival (moderate HR 1.59. 95\% CI 1.16-2.18, $p=0.04$; severe HR $1.83,95 \%$ CI $1.22-2.72, p=0.003)$ and bladder cancer specific survival (moderate HR 1.50, 95\% CI $1.04-2.15, p=0.028$; severe HR $1.65,95 \%$ CI $1.04-2.62, p=0.034)$ even after controlling for age, adjuvant chemotherapy, surgeon procedure volume, pathologic $\mathrm{T}$ stage, pathologic lymph node status, total number of lymph nodes removed, surgical margin status and lymphovascular invasion [34]. A recent SEER analysis demonstrated that grade and stage were associated with non-bladder cancer associated death amongst patients diagnosed with bladder cancer, however the grade/stage categories played a much greater role in bladder cancer specific mortality, suggesting that the main cause of death for patients with muscle invasive disease is bladder cancer and not a patient's associated co-morbidities [35]. The findings by other authors that patients with increased co-morbidities have worse overall survival likely reflect that patients with more co-morbidities may be less likely to undergo a radical cystectomy for treatment and may have fewer and less-effective options available for neo-adjuvant and adjuvant therapy.

\section{Limitations}

There are several limitations to our study. SEER data does not provide specific information on diagnosis, neo-adjuvant or adjuvant treatment, or follow-up. Cancer stage II \& III is summed together in this analysis and SEER does not include information on margin status and only includes nodal status data since 2004, so we did not include it in our analysis. In addition, there is little information on patient co-morbidities and other factors which have shown to impact cystectomy outcomes, such as the type of hospital (comprehensive cancer center vs. academic vs private vs VA hospital) in which the surgery took place or the hospital volume. Our analysis only includes patients who had undergone a cystectomy, which also means that this population was healthy enough to obtain pre-operative clearance to undergo the surgery and thus may reflect a population with fewer and/or less severe co-morbidities. The factors mentioned above have been shown to affect bladder cancer survival after cystectomy. This information could have provided additional information on which type of patient is more likely to die with and of blad- der cancer and why African American patients have worse all-cause mortality. In addition, the SEER program has expanded in geography over time, which means that any differences observed could actually reflect socioeconomic changes and not real differences. However, our analysis controlled for year of diagnosis, which decreases some of this variability in the data. Lastly, this analysis has the limitations inherent in a retrospective review, including this being a select population for which a cystectomy was chosen to treat bladder cancer and thus we are unable to control for individual physician decision making on who received a cystectomy. Given the data limitations, additional analyses are required to provide more specific information as to why the differences exist.

Although there are several limitations, this is the only analysis aimed at determining if African American/Caucasian racial differences exist in all-cause and cancer-specific mortality for patients who underwent a cystectomy for the treatment of bladder cancer and provides data on what factors may contribute to differences in survival. The analysis also uses a matching methodology, which further decreases variability between the cohorts.

\section{CONCLUSION}

African American patients who undergo a cystectomy for bladder cancer have worse all-cause and cancer-specific survival than Caucasian patients. However, after controlling for sex, age, year of diagnosis, marital status, region of treatment, and stage at diagnosis, African Americans who undergo a cystectomy no longer have worse bladder cancer-specific mortality than Caucasians, but disparities remain in overall mortality. Differences in age, sex, and stage at diagnosis explain some, but not all of the differences in survival. These data suggest that patients who get a cystectomy for bladder cancer African American patients may be dying from causes not directly related to the diagnosis, biology, and management of their bladder cancer. Additional analyses are required to provide further information on why African American patients with bladder cancer have worse overall survival then similar Caucasian patients.

\section{CONFLICT OF INTEREST}

The authors have no conflict of interest to report. 


\section{REFERENCES}

[1] American Cancer Society. Atlanta: The Society; 2015; cited 2015 Mar 15]. Available from http://www.cancer.org/ cancer/bladdercancer/detailedguide/bladder-cancer-keystatistics

[2] Burger M, Catto JW, Dalbagni G, Grossman HB, Herr H, Karakiewicz P, Kassouf W, Kiemeney LA, La Vecchia C, Shariat S, Lotan Y. Epidemiology and risk factors of urothelial bladder cancer. Eur Urol 2013;63(2):234-41.

[3] Mallin K, David KA, Carroll PR, Milowsky MI, Nanus DM. Transitional cell carcinoma of the bladder: Racial and gender disparities in survival (1993 to 2002), stage and grade (1993 to 2007). J Urol 2011;185(5):1631-6.

[4] Brookfield, KF, et al. Survival disparities among African American women with invasive bladder cancer in Florida. Cancer 2009;115:4196-209.

[5] Underwood W III DR, Williams C, Lee CT. Gender and geographic influence on the racial disparity in bladder cancer mortality in the US. Journal of the American College of Surgeons 2006;202:284-90.

[6] Scosyrev E, Noyes K, Feng C, Messing E. Sex and racial differences in bladder cancer presentation and mortality in the US. Cancer 2009;115(1):68-74.

[7] Yee DS, Ishill NM, Lowrance WT, Herr HW, Elkin EB. Ethinic differences in bladder cancer survival. Urology 2011;78:544-49.

[8] Howard J, Hankey BF, Greenberg RS, Austin DF, Correa P, Chen VW, Durako S. A collaboratve study of differences in the survival rates of African American patients and Caucasian patients with cancer. Cancer 1992;69(9):2349-60

[9] Scosyrev E, Noyes K, Feng C, Messing E. Sex and racial differences in bladder cancer presentation and mortality in the US. Cancer 2009;115(1):68-74.

[10] Blackwell M, Iacus G, King G, Porro G. Cem: Coarsened exact matching in Stata. Stata J 2009;9(4):524-46.

[11] Hankey BF, Myers MH. African American/Caucasian differences in bladder cancer patient survival. J Chronic Dis 1987;40(1):65-73.

[12] Yee DS, Ishill NM, Lowrance WT, Herr HW, Elkin EB. Ethnic differences in bladder cancer survival. Urology 2011;78(3):544-9.

[13] Prout GR Jr, Wesley MN, Greenberg RS, Chen VW, Brown CC, Miller AW, Weinstein RS, Robboy SJ, Haynes MA, African Americanlow RS, Edwards BK. Bladder cancer: Race differences in extent of disease at diagnosis. Cancer 2000;89(6):1349-58.

[14] Prout GR Jr, Wesley MN, McCarron PG, Chen VW, Greenberg RS, Mayberry RM, Edwards BK. Survival experience of African American patients and Caucasian patients with bladder carcinoma. Cancer 2004;100(3):621-30.

[15] Lee CT, Dunn RL, Williams C, Underwood W 3rd. Racial disparity in bladder cancer: Trends in tumor presentation at diagnosis. J Urol 2006;176(3):927-33; discussion 933-4.

[16] Krongrad A, Lai H, Burke MA, Goodkin K, Lai S. Marriage and mortality in prostate cancer. J Urol 1996;156(5):169670 .

[17] Gore JL, Kwan L, Saigal CS, Litwin MS. Marriage and mortality in bladder carcinoma. Cancer 2005;104(6):118894.

[18] Johnson NJ, Backlund E, Sorlie PD, Loveless CA. Marital status and mortality: The national longitudinal mortality study. Ann Epidemiol 2000;10(4):224-38.
[19] Datta GD, Neville BA, Kawachi I, Datta NS, Earle CC Marital status and survival following bladder cancer. J Epidemiol Community Health 2009;63(10):807-13.

[20] Nelles JL, Joseph SA, Konety BR. The impact of marriage on bladder cancer mortality. Urol Oncol 2009;27(3):263-7

[21] Noon AP, Albertsen PC, Thomas F, Rosario DJ, Catto JW. Competing mortality in patients diagnosed with bladder cancer: Evidence of undertreatment in the elderly and female patients. Br J Cancer 2013;108(7):1534-40.

[22] Choo MS, Jeong CW2, Kwak C2, Kim HH2, Ku JH. Effect of sex on prognosis of urothelial carcinoma: Propensity score matching analysis. Clin Genitourin.

[23] Jamal A, Homa D, O'Connor E, Babb SD, Caraballo RS, Singh T, Hu SS, King BA. Current cigarette smoking among adults - United States, 2005-2014. CDC Morbidity and Mortality Weekly Report 2015;64(44):1233-40

[24] Moon-Howard J. African American women and smoking: Starting later. Am J Public Health 2003;93(3):418-20.

[25] Mayer WJ, McWhorter WP. African American/Caucasian differences in non-treatment of bladder cancer patients and implications for survival. Am $\mathrm{J}$ Public Health 1989;79(6):772-5

[26] Konety BR, Joslyn SA. Factors influencing aggressive therapy for bladder cancer: An analysis of data from the SEER program. J Urol 2003;170(5):1765-71.

[27] Hollenbeck BK, Dunn RL, Ye Z, Hollingsworth JM, Lee CT, Birkmeyer JD. Racial differences in treatment and outcomes among patients with early stage bladder cancer. Cancer 2010;116(1):50-6.

[28] Casey MF, Gross T, Wisnivesky Jm Stensland KDm Oh WK, Galsky. The impact of regionalization of cystectomy on racial disparities in bladder cancer care. J Urol 2015; 194(1):36-41.

[29] Barocas DA, Alvarez J, Koyama T, Anderson CB, Gray DT, Fowke JH, You C, Chang SS, Cookson MS, Smith JA Jr, Penson DF. Racial variation in the quality of surgical care for bladder cancer. Cancer 2014;120(7):1018-25.

[30] Konety BR, Allareddy V, Carroll PR. Factors affecting outcomes after radical cystectomy in African Americans. Cancer 2007;109(3):542-8.

[31] Taub DA, Hollenbeck BK, Cooper KL, Dunn RL, Miller DC, Taylor JM, Wei JT. Racial disparities in resource utilization for cystectomy. Urology 2006;67(2):288-93.

[32] Malmström PU, Busch C, Norlén BJ. Recurrence, progression and survival in bladder cancer. A retrospective analysis of 232 patients with greater than or equal to 5 -year followup. Scand J Urol Nephrol 1987;21(3):185-95.

[33] Lund L, Jacobsen J, Clark P, Borre M, Nørgaard M. Northern Danish Cancer Quality Assessment Group. Impact of comorbidity on survival of invasive bladder cancer patients, 1996-2007: A Danish population-based cohort study. Urology 2010;75(2):393-8.

[34] Fairey AS, Jacobsen NE, Chetner MP, Mador DR, Metcalfe JB, Moore RB, Rourke KF, Todd GT, Venner PM, Voaklander DC, Estey EP. Associations between comorbidity, and overall survival and bladder cancer specific survival after radical cystectomy: Results from the Alberta Urology Institute Radical Cystectomy database. J Urol 2009;182(1):85-92.

[35] Scosyrev E, Wu G, Golijanin D, Messing E. Non-bladder cancer mortality in patients with urothelial cancer of the bladder. Urol Oncol 2013;31(5):656-63. 